\title{
Utilidad de la Colagenasa en Procesos Inflamatorios de Tipo Ulcerativo o Erosionado del Cuello Uterino
}

\author{
Dr. Carlos Villamizar Martín*
}

\section{Introducción}

En la consulta ginecológica se encuentran frecuentemente lesiones inflamatorias del cuello uterino, que se denominan cervicitis, las cuales se clasifican en agudas o crónicas, pudiendo ser de tipo ulcerativo o erosionado. Se tienen como causas principalmente: traumatismo del parto, tratamiento ginecológico instrumental, hiperestrinismo, hipoestrinismo, coito, secreción excesiva de las glándulas cervicales, alcalinidad alta del moco cervical.

Para el tratamiento de estas lesiones se han empleado diversidad de métodos locales, tópicos (anti-inflamatorios, sustancias quelantes, cauterización y criocirugía).

Llama la atención una sustancia enzimática, la Colagenasa**, cuyas características químicas permiten activar el colágeno nativo de los tejidos, rompiendo las cadenas de aminoácidos en las zonas ricas en prolina. La Colagenasa de

MD. Ginecólogo dei I. S. S.

MD. Adjunto Hospitalario IMI.

* $\quad$ Iruxol Simplex Unguiento Knoll mayor actividad es la procedente del Clostridium Histolyticun, la cual tiene una fracción, la Clostridium Peptidasa A, que contiene escasa proporción de hialuronidasa, lecitinasa $y$ emolisina $y$ un índice de proteasas y peptidasas más elevado, lo cual favorece la limpieza de las heridas. Esta sustancia hasta el momento se había empleado para facilitar la granulación, la epitelización y la optima cicatrización de heridas dérmicas. Por su acción farmacológica, se quiso comprobar su efectividad en el tratamiento de procesos ulcerativos o erosionados del cuello uterino, a pesar de que en la literatura mundial no se encuentra ningún trabajo en donde se mencione su empleo para esta indicación.

\section{Material y Métodos}

Se estudiaron 50 pacientes ambulatorias de la consulta externa, institucional y privada, que al exámen clínico presentaban signos de ulceración o erosión del cuello uterino.

La edad de las pacientes osciló entre los 20 y los 43 años, siendo las edades más frecuentes las de 26,28 y 30 años. (Cuadro No. 1). 
Cuadro No. 1

\section{DISTRIBUCION POR GRUPOS DE EDAD Y NUMERO DE PACIENTES}

\begin{tabular}{|c|c|c|}
\hline $\begin{array}{c}\text { Grupos } \\
\text { de edad }\end{array}$ & $\begin{array}{c}\text { No. } \\
\text { Pacientes }\end{array}$ & Porcentaje \\
\hline $20-24$ años & 7 & $14 \%$ \\
$25-29$ & 21 & $42 \%$ \\
$30-34$ & 11 & $22 \%$ \\
$35-39$ & 6 & $12 \%$ \\
$40-44$ & 5 & $10 \%$ \\
\hline Totales & 50 & $100 \%$ \\
\hline
\end{tabular}

La sintomatología había comenzado de dos meses a un año antes del presente estudio, habiendo recibido el $28 \%$ de las pacientes tratamiento local previo.

Se empleó la Colagenasa en lesiones cervicales de tipo ulcerativo en 27 pacientes y de tipo erosionado en 23 (Cuadro No. 2).
Cuadro No. 2

\section{EMPLEO DE LA COLAGENASA SEGUN GRUPOS DE EDAD Y LESIONES}

\begin{tabular}{|c|c|c|}
\hline $\begin{array}{c}\text { Grupos } \\
\text { de edad }\end{array}$ & Ulceraciones & Erosiones \\
\hline $20-24$ años & $2-4 \%$ & $6-12 \%$ \\
$25-29$ & $17-34 \%$ & $7-14 \%$ \\
$30-34$ & $2-4 \%$ & $5-10 \%$ \\
$35-39$ & $4-8 \%$ & $2-4 \%$ \\
$40-44$ & $2-4 \%$ & $3-6 \%$ \\
\hline Totales & $27-54 \%$ & $23-46 \%$ \\
\hline
\end{tabular}

Se requería que las pacientes tuvieran citología cervicovaginal oncológica previa, habiéndose encontrado 2 casos con citología Grado III, de Papanicolau.

El tratamiento se hizo empleando la Colagenasa sola en 25 casos indiscrimi-

Cuadro No. 3

EMPLEO DE LA COLAGENASA, SEGUN GRUPOS DE EDAD, TIPO DE LESION Y ASOCIACION

\begin{tabular}{|c|c|c|c|c|c|c|}
\hline \multirow{2}{*}{$\begin{array}{l}\text { Grupos } \\
\text { de edad }\end{array}$} & \multicolumn{2}{|c|}{$\begin{array}{c}\text { Colagenasa } \\
\text { Sola }\end{array}$} & \multicolumn{2}{|c|}{$\begin{array}{c}\text { Colagenasa }+ \\
\text { Antibiótico }\end{array}$} & \multicolumn{2}{|c|}{$\begin{array}{c}\text { Colagenasa + } \\
\text { Trim. Sulfa }\end{array}$} \\
\hline & Ulceración & Erosión & Ulceración & Erosión & Ulceración & Erosión \\
\hline $20-24$ & 1 & 5 & 1 & - & - & 1 \\
\hline $25-29$ & 5 & 4 & 7 & - & 4 & 3 \\
\hline $30-34$ & 2 & 4 & 1 & - & - & 1 \\
\hline $35-39$ & 2 & - & 1 & 1 & 1 & 1 \\
\hline $40-44$ & - & 2 & 2 & - & - & 1 \\
\hline Totales & 10 & 15 & 12 & 1 & 5 & 7 \\
\hline
\end{tabular}


nadamente: 10 con ulceración cervical y 15 con erosión cervical.

Se empleó la Colagenasa asociada a antibióticos por vía oral (Tetraciclina cápsulas de 250 mgr. cada 12 horas), por presentar las lesiones concomitancia con secreción purulenta de olor fétido, así: 12 casos con ulceración y 1 con erosión cervical; para un total de 13 casos. Se empleó la colagenasa asociando trimethoprim-sulfa (comprimidos de 500 mgr. cada 12 horas), en lesiones que presentaban secreción purulenta no fétida asi: 5 con ulceración y 7 con erosión cervical, para un total de 12 casos. (Cuadros Nos. 3, 4 y Gráfica No. 1).

Se tuvo el siguiente parámetro para evaluar la respuesta:

Excelente, cuando la curación fue total.
Buena, cuando la mejoría de la lesión fue sustancial.

Regular, cuando la limpieza de la lesión fue parcial y no se consiguió mejoría después de 5 semanas de tratamiento.

Para objetivar el resultado se tomo fotografía antes, durante y después del tratamiento.

Técnica de aplicación. Se efectúa la especuloscopia, centralizando el cuello uterino, se limpia el mismo con una torunda de algodón empapada con suero fisiológico y se aplica la colagenasa mediante el uso de un aplicador de fácil diseño. La aplicación se efectuó dos veces por semana, excepto cuando las pacientes se encontraban con la menstruación.

Gráfica No. 1

\section{EMPLEO DE LA COLAGENASA SEGUN NUMERO DE PACIENTES, TIPO DE LESION Y ASOCIACIONES}

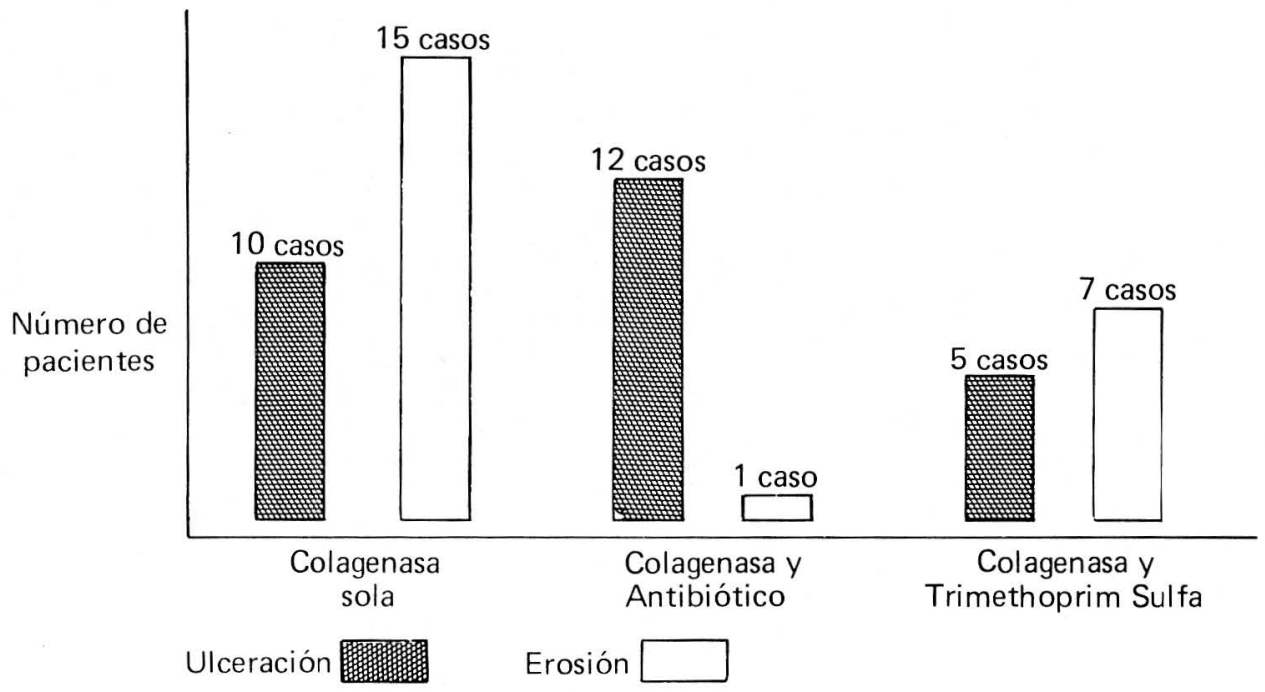




\section{Resultados}

En el presente estudio se obtuvo evolución excelente en 38 casos $(76 \%)$, buena en 9 casos $(18 \%)$ y regular en 3 casos $(6 \%)$. (Cuadro No. 5 y Gráfica No. 2).

De los casos en que se empleo la Colagenasa sola se obtuvo evolución excelente en 23, buena en 2; cuando se empleó la Colagenasa asociándola con antibióticos se obtuvo evolución excelente en 4 casos, buena en 6 casos $y$ regular en 3 casos y cuando se empleó asociándola con trimethoprim-sulfa, el resultado de la evolución fue excelente en 11 casos y buena en 1 caso. (Cuadro No. 6 y Gráfica No. 3).

De los casos en los que se empleó la Colagenasa asociada al antibiótico (12 con ulceración y 1 con erosión), hubo necesidad de efectuar cauterización cervical en 9, por presentar ulceración crónica que no mejoraba después de 15 días de tratamiento; sin embargo, después de la cauterización se continuó el mismo, consiguiéndose evolución buena en 6 casos y regular en 3 casos.
Gráfica No. 2

\section{RESULTADO DEL TRATAMIENTO SEGUN NUMERO DE PACIENTES Y TIPO DE LESION}

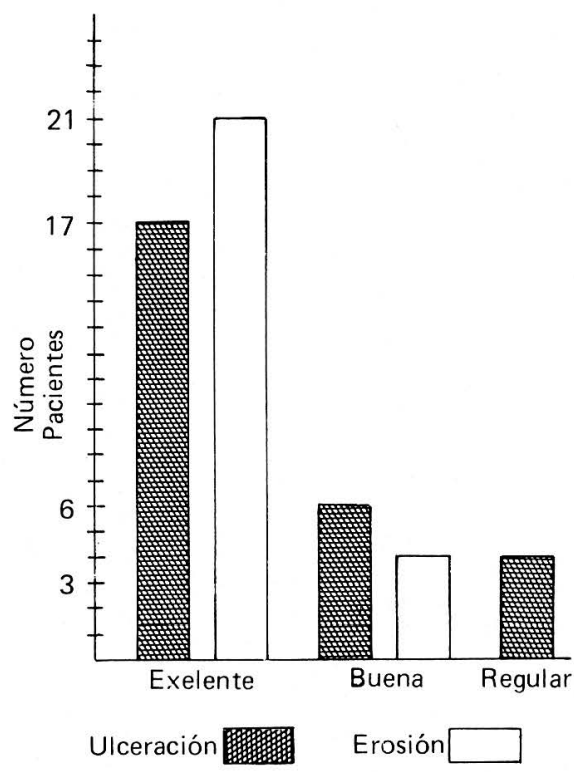

Cuadro No. 4

DISTRIBUCION POR EDAD SEGUN EL TRATAMIENTO

\begin{tabular}{|c|c|c|c|c|}
\hline Grup. Edad & $\begin{array}{c}\text { Pacientes con } \\
\text { Colagenasa Sola }\end{array}$ & $\begin{array}{c}\text { Con Colagenasa } \\
\text { y Antibiótico }\end{array}$ & $\begin{array}{c}\text { Colagenasa } \\
\text { Trim. Sulfa }\end{array}$ & Totales \\
\hline $20-24$ & 6 & 1 & 1 & 8 \\
$25-29$ & 9 & 7 & 7 & 23 \\
$30-34$ & 6 & 1 & 1 & 8 \\
$35-39$ & 2 & 2 & 2 & 6 \\
$40-44$ & 2 & 2 & 1 & 5 \\
\hline Totales & $25=50 \%$ & $13=26 \%$ & $12=24 \%$ & $50=100 \%$ \\
\hline
\end{tabular}


El promedio de aplicaciones por paciente fue de 5 y el de días de tratamiento fue de 18 , con los resultadios anotados anteriormente.

Los porcentajes de evolución fueron entre excelentes $y$ buenos el $94 \%$ (47 pacientes) y regular $6 \%$ (3 pacientes)

Durante el tratamiento no se observó exacervación del proceso ni se presentaron reacciones alérgicas a la droga en estudio.

Los dos casos que presentaban citología Grado III cambiaron después del tratamiento a citología Grado II en razón a la mejoría del proceso inflamatorio que presentaron las pacientes.

Se observó que una vez finalizado el estudio, 3 pacientes quedaron embarazadas; dichas pacientes se encontraban en estudio de esterilidad, demostrándose que una de las causas de ésta, es el factor cervical.

\section{Resumen y Conclusiones}

Se efectuó el trabajo en busca de innovación para el tratamiento de lesio-
Gráfica No. 3
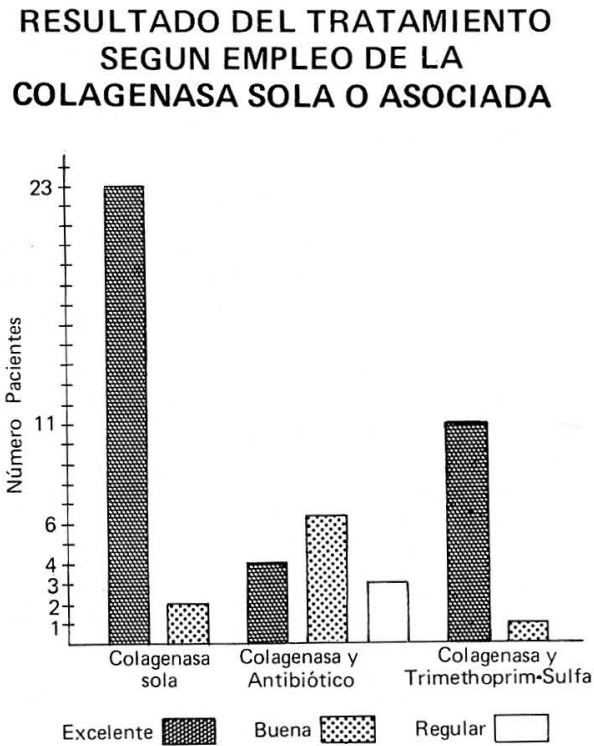

nes inflamatorias del cuello uterino de tipo ulcerativo o erosionado, empleando la colagenasa para facilitar la cicatrización.

Cuadro No. 5

RESULTADO DEL TRATAMIENTO

SEGUN EDAD Y TIPO DE LESION

\begin{tabular}{|c|c|c|c|c|c|c|}
\hline \multirow{2}{*}{ Edad } & \multicolumn{2}{|c|}{ Excelente } & \multicolumn{2}{c|}{ Buena } & \multicolumn{2}{c|}{ Regular } \\
\cline { 2 - 7 } & Ulceración & Erosión & Ulceración & Erosión & Ulceración & Erosión \\
\hline $20-24$ & 2 & 5 & - & - & - & - \\
$25-29$ & 9 & 6 & 3 & 2 & 3 & - \\
$30-34$ & 3 & 5 & - & 1 & - & - \\
$35-39$ & 3 & 3 & 1 & - & - & - \\
$40-44$ & - & 2 & 2 & - & - & - \\
\hline Totales & 17 & 21 & 6 & 3 & 3 & - \\
\hline
\end{tabular}


Cuadro No. 6 RESULTADO DEL TRATAMIENTO Y PORCENTAJE SEGUN EMPLEO
DE LA COLAGENASA SOLA O ASOCIADA

\begin{tabular}{|l|c|c|c|cc|}
\hline Resultado & $\begin{array}{c}\text { Colagenasa } \\
\text { sola }\end{array}$ & $\begin{array}{c}\text { Colagenasa y } \\
\text { antibiótico }\end{array}$ & $\begin{array}{c}\text { Colagenasa } \\
\text { Trim. Sulfa }\end{array}$ & $\begin{array}{c}\text { Porcentaje y } \\
\text { No. total de casos }\end{array}$ \\
\hline Excelente & 23 & 4 & 11 & $76 \%$ & $(38 ;$ \\
Buena & 2 & 6 & 1 & $18 \%$ & $(8)$ \\
Regular & - & 3 & - & $6 \%$ & $(3)$ \\
\hline Totales & 25 & 13 & 12 & $100 \%$ & \\
\hline
\end{tabular}

Por los resultados obtenidos el tiempo de tratamiento con Colagenasa no fue tan prolongado y la cantidad de aplicaciones no fue numerosa a pesar de haberse empleado principalmente en lesiones crónicas y que un $28 \%$ de las pacientes estudiadas habían recibido tratamiento previo al estudio con los métodos tradicionales.

La aplicación de la Colagenasa sobre la lesión debe ser hecha por el médico tratante requiriéndose para esto constancia por parte de la paciente.
Por los buenos resultados conseguidos $(94 \%)$ se ha demostrado que la Colagenasa es de gran utilidad para el desbridamiento del tejido lesionado en procesos ulcerativos y erosionados del cuello uterino, razón por la cual se recomienda su uso para el tratamiento de estas lesiones en Ginecología.

Nọo se observó reacción alérgica, ni exacervación del proceso en ningún caso.

\section{UTILITY OF COLAGENASA IN INFLAMMATORY PROCESSES IN CASES OF ULCERATION OR EROSION OF THE CERVIX}

\section{Summary and conclusions}

The work was undertaken in search of innovations in the treatment of inflammatory lesions, in cases of ulceration or erosion of the cerviz, using Colagenasa to facilitate the healing.

According to the results, the length of treatment was not prolonged, nor were the applications numerous. This, despite the fact that it was used principally on chronic lesions, and that $28 \%$ of the patients studied had previously been treated with traditional methods.

The application of Colagenasa on the lesion must be done by the doctor in charge, requiring attendance on the part of the patient.

The results show that in $94 \%$ of the cases, Colagenasa is of great utility in the separation of lesion tissue in cases of 
ulceration and erosion of the cervix. For this reason, it is recoinmended for the treatment of these lesions in gynecology.

\section{Bibliografía}

1. BARRETT D. KLIBANSKI A; Desbridamiento con Colagenasa AM. J. Nurs, 73: 849-51 Mayo 73.

2. ALVES D' ASSU MPCAO E: Desbridamiento químico por la Colagenasa, Rev. Bras. Cir. 66-1976.

3. SHERRY S. FLETCHER AP; Enzimas proteolíticas, una evaluación terapéutica. Clin. Pharmacol Ther 1: 202, 1960.
No allergic reaction nor deterioration of the condition was observed in any of the cases.

4. P. BALLESTEROS GARCIA E I. MARTIN GARCIA: Tratamiento con Colagenasa de las lesiones dérmicas subsecuentes a la terapéutica quirúrgica o radiológica de pacientes oncológicos.

Med. Clin. Clínica Médica Años XIX No. 213, mayo 1979 , págs 38 a 45 . 